\title{
Héctor Mario Pucciarelli Un maestro de la antropología biológica
}

\section{María Antonia Luis}

\author{
Laboratorio de Investigaciones en Ontogenia y Adaptación (LINOA). Facultad de Ciencias Naturales y \\ Museo (FCNyM), Universidad Nacional de La Plata (UNLP). La Plata, Buenos Aires, Argentina \\ ORCID: 0000-0001-9601-5490 \\ Correo electrónico: maluis@fcnym.unlp.edu.ar

\section{Evelia Edith Oyhenart} \\ Laboratorio de Investigaciones en Ontogenia y Adaptación (LINOA). Facultad de Ciencias Naturales y \\ Museo (FCNyM), Universidad Nacional de La Plata (UNLP). La Plata, Buenos Aires, Argentina. \\ Instituto de Genética Veterinaria (IGEVET)-CCT La Plata, CONICET. Facultad de Ciencias Veterinarias \\ (FCV). Universidad Nacional de La Plata (UNLP). La Plata, Buenos Aires, Argentina. \\ ORCID: 00oo-0003-1331-7368 \\ Correo electrónico: oyhenart@fcnym.unlp.edu.ar
}

El 27 de noviembre pasado murió Héctor Mario Pucciarelli. La noticia de su partida causó tristeza, que bien pronto ha tornado en una cierta desolación. Sin embargo, sería injusto recordar a Héctor con tristeza o desolación ya que supo, con su personal trayectoria, llenar buena parte de la historia reciente de la Antropología Biológica en la Universidad Nacional de La Plata.

Héctor era una persona inquieta, ávida de ilusiones relacionadas con la investigación y la ciencia, a las que dedicó su vida. Necesitaba vislumbrar nuevos horizontes que atrajeran su curiosidad y su preocupación. Por ello, su recuerdo merece ser pleno de reconocimiento y alegría, porque su figura fue creciendo, fruto de su tenacidad consecuente y, como decía el pensador medieval Bernardo de Chartres, "sobre sus hombros se subieron los discípulos para ver más lejos y seguir avanzando en la tarea inacabable de explorar la realidad." (en Merton, 1990, p. 97).

Nació en La Plata el 20 de junio de 1939. En 1967 egresó de la Facultad de Ciencias Naturales y Museo de la UNLP (FCNyM) con el título de licenciado en Antropología, y en 1971 - cuando obtuvo en la defensa de su tesis la calificación de sobresaliente con recomendación unánime de publicaciónalcanzó el grado de doctor en Ciencias Naturales. Se formó como antropólogo en el contexto de un plan de estudios, el de 1958, que reflejaba el paradigma de simplificación y disyunción, basado en la reducción y separación de los saberes, en el que la antropología biológica estaba representada solo por dos asignaturas. Pronto comprendió que dicha planificación no era el camino para

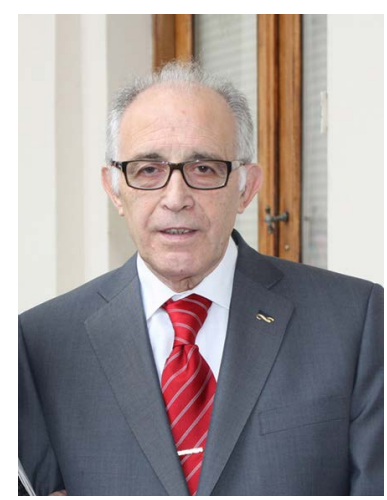

Fig. 1. Héctor Mario Pucciarelli

Nota de los editores: Héctor Mario Pucciarelli, fue miembro del Comité Académico Asesor de RUNA en el área de Antropología Biológica entre los años 2009 y 2017, su partida ha sido una perdida importante para nuestra revista. Sin embargo, nos queda el honor de haber contado con sus aportes, comentarios y sugerencias durante todos esos años. Vaya esta nota de quienes compartieron su vida y su trabajo, como nuestro homenaje. 
alcanzar la formación de antropólogos capaces de abordar las problemáticas planteadas en la disciplina a nivel mundial. Junto con otros colegas, en especial con su amigo Raúl Carnese, comenzaron a promover la necesidad de discutir un nuevo plan de estudios, que finalmente se concretó en la versión de 1966, en el que se distinguen tres orientaciones: Antropología Social, Arqueología y Antropología Biológica, con la característica de la especialización temprana. Sin dudas, alentaba en él la potencia del nuevo paradigma, el de la complejidad, cuyo objetivo es alentar la articulación y la contextualización de lo biológico, lo sociocultural y lo arqueológico en una conjugación dialéctica que permitiera explicar la condición humana. Más tarde, en el plan de estudios de Antropología de 1985, aún vigente, Héctor incorporó algunas de sus conceptualizaciones teóricas, que marcaron una ruptura epistemológica respecto de los planes antes mencionados. Allí, integró en la formación de los antropólogos las dimensiones biológica y cultural, en una síntesis dialéctica que permite explicar la variabilidad biológica onto y filogenética.

No es tarea sencilla resumir la diversidad de aspectos que asumió su trayectoria. En ese sentido, con referencia a su tarea docente de formación de grado, en la FCNyM-UNLP, en 1965, comenzó como ayudante alumno en la cátedra de Antropología General. Fue jefe de Trabajos Prácticos en la misma cátedra y profesor titular en Biología Humana. En 1983, una vez recuperada la democracia, accedió al cargo de profesor titular ordinario en la cátedra de Antropología Biológica I. Su rol docente en la enseñanza de posgrado se reveló a partir del dictado de cursos y seminarios en los que supo abordar los temas centrales de la antropología biológica desde una perspectiva evolutiva e interdisciplinaria. Es grato al espíritu recordarlo dictando sus clases, en las que, indefectiblemente, con pasión, promovía el debate productivo basado en los tres pasos de la dialéctica hegeliana: la tesis con sus contradicciones intrínsecas, la antítesis que es contradictoria con la primera y la síntesis superadora, la que, a su vez, será una nueva tesis en la dinámica evolutiva. En ese sentido, son inolvidables, para quienes fuimos estudiantes durante el oscuro período de la dictadura, las clases que Héctor dictó sobre evolución humana, por explícito pedido nuestro En dos sábados, desde la mañana hasta bien entrada la tarde, supo interpretar plenamente nuestra orfandad sobre el tema, con un esfuerzo en el que combinó conocimientos, análisis y síntesis. Y tal vez, para aliviar tamaño desgaste de concentración, ensayaba dos formas de aliviar tanta tensión: por momentos, se detenía frente al pizarrón y, apoyándose sobre el "puntero" de madera, se balanceaba rítmicamente; o bien, fumaba su pipa con fruición y recorría, una y otra vez, el pasillo estrecho que dejábamos los estudiantes sentados en el piso del aula. Esa labor no solo es reconocida por quienes fuimos sus discípulos; también a propuesta de la FCNyM, la Universidad Nacional de La Plata lo designó Profesor Extraordinario, en la categoría de Emérito, en función de sus condiciones sobresalientes e integridad ético-moral observadas en su trayectoria profesional.

Asimismo, su trayectoria en investigación también fue pletórica de innovaciones. Se inició en la FCNyM como lugar de trabajo, para cumplir una beca de iniciación a la Actividad Creadora, otorgada por la Universidad Nacional de La Plata, con el Tema: "Alteraciones de la Morfología Craneana en Grupos del Noroeste Argentino por Posible Etiología Genético-Ambiental", en el período 1967-1969. Continuó con una beca de perfeccionamiento otorgada por la Comisión de Investigaciones Científicas de la Provincia de Buenos Aires (CICPBA), con el tema: "Estudio Experimental Sobre Alteraciones en la Morfología del Neurocráneo", con lugar de trabajo en el Instituto de Neurobiología, en el período 1970-1972, y como investigador, hasta 
1975 permaneció en la CIC. En ese año ingresó en el Consejo Nacional de Investigaciones Científicas y Técnicas (CONICET), donde alcanzó la categoría de Investigador Superior, máximo grado para esa institución.

Sus investigaciones se caracterizaron por la introducción de innovaciones teóricas y metodológicas derivadas de su conceptualización de la antropología biológica como una ciencia que se ocupa de procesos resultantes de la interacción genético-ambiental y no de hechos aislados. Ello implicó el reemplazo del concepto tipológico-descriptivo por un enfoque analítico poblacional y, en lo metodológico, la introducción del método experimental. Este "nuevo" enfoque permitió estudiar experimentalmente el modo en que los factores ambientales actúan sobre los patrones morfogenéticos, sin que ello significase abandonar el marco teórico propio de la antropología.

Su trabajo "Experimental technique for cranial deformation in growing rats", publicado en 1973 (Pucciarelli, 1973), fue el primer estudio de antropología biológica experimental realizado en nuestro país. Esa técnica fue aplicada con posterioridad en investigaciones con diferentes objetivos, tales como estimar la influencia de la práctica cultural de la deformación craneana en la persistencia de huesos craneanos supernumerarios o estudiar los efectos de la nutrición sobre el crecimiento craneofacial. Es preciso recordar, en este punto, que gracias a una beca externa viajó a la Universidad de Columbia, donde conoció al Dr. Melvin L. Moss, con quien trabajó. Ese vínculo fue trascendente en la labor de Héctor, tal como él mismo lo expresara en ocasión de realizar el obituario de Moss para la Revista Argentina de Antropología Biológica en 2006, donde afirmaba que
la línea de razonamiento experimental en Antropología Biológica, fundada por el inolvidable profesor Washburn, no había muerto sino que con la claridad conceptual de Mel, tomó nuevo ímpetu y se cristalizó en su famoso trabajo experimental realizado en 1960 junto con Young. Allí se logró cimentar, entre otras cosas, la idea de aplicar el método de los Elementos Finitos a la práctica Antropobiológica, que llegó a su coronación con la magnífica idea de Mel de combinar ese método con su concepto de matriz funcional, lo cual terminó por provocar un giro teórico fundamental en la concepción del cráneo como un complejo óseo, en el que cada componente crece y se transforma en función de su propia dinámica interna y de las demandas funcionales de sus componentes vecinos (Pucciarelli, 2006).

Puede afirmarse que Héctor comprendió la enorme importancia y la necesidad de recurrir a la experimentación cuando la metodología comparativa resultaba ineficaz en la consecución de un resultado aceptable o al momento de dirimir entre dos o varias hipótesis contrapuestas para explicar el mismo problema.

El Centro de Investigaciones en Genética Básica y Aplicada (CIGEBA), en la actualidad Instituto de Genética Veterinaria (IGEVET) (FCV-UNLP-CONICET) fue, desde 1991 hasta 1999, el espacio donde cumplió funciones de subdirector y donde desarrolló tres ejes temáticos que abordaron la variación craneana métrica y no métrica y el dimorfismo sexual, en respuesta a diferentes tipos de desnutrición aplicados a animales de laboratorio.

Es preciso destacar que, para Héctor, ninguno de los temas nodales de la antropología biológica le fueron ajenos, como tampoco la necesidad de un abordaje interdisciplinario. En este sentido, a partir de 1983, con la recuperación de la democracia en nuestro país, se iniciaron los contactos entre los equipos de investigación de la Universidad de Buenos Aires, bajo la dirección 
de Raúl Carnese; de la UNLP a cargo de Héctor; del Hospital de Niños "Sor María Ludovica", representado por Luis Guimarey; y del Centro Nacional Patagónico (CENPAT-CONICET) mediante Silvia Dahinten. Se plantearon el objetivo de realizar investigaciones sobre el crecimiento y dimorfismo sexual en poblaciones cosmopolitas y aborígenes. Sin lugar a dudas, el estudio del crecimiento humano mereció especial interés, por tratarse de un proceso que permite abordar la compleja interacción entre factores biológicos, culturales y ambientales. Uno de los primeros trabajos fue realizado en escolares de Villa IAPI (Quilmes, Provincia de Buenos Aires), entre 1988 y 1990, una barriada pobre, similar a las muchas ubicadas en la periferia de las ciudades argentinas más populosas. Un ambiente socioeconómico caracterizado por el trabajo temporario de los hombres - en alternancia con períodos prolongados de desempleo o subempleo- con las mujeres ocupadas de las tareas domésticas. La situación ambiental era igualmente precaria, con ausencia de agua potable domiciliaria y de adecuadas instalaciones para la eliminación de excretas y aguas servidas. La dieta de esta población revelaba un alto contenido de lípidos y glúcidos, y era baja en proteínas y fibras vegetales. Por cierto, los resultados dieron cuenta de ese escenario de vulnerabilidad por cuanto revelaron que los niños de Villa IAPI tenían retraso del crecimiento lineal, a expensas del acortamiento de los miembros inferiores, disminución de la masa muscular y aumento del panículo adiposo. También el dimorfismo sexual acusó modificaciones relacionadas con ese escenario de carencias.

Con respecto a los estudios realizados en poblaciones aborígenes, merece citarse el que se llevó a cabo en la población infantil mapuche de Río Negro, cuyos resultados constituyeron la única referencia de crecimiento, aún vigente, para Argentina (Carnese et al., 1994). El equipo formado por investigadores de la UBA, el CENPAT y la UNLP continuó, a partir de 1996, trabajando con una de las tres poblaciones tehuelche, asentada en la reserva El Chalía, al sudoeste de la provincia de Chubut. El objetivo planteado fue analizar el crecimiento, el dimorfismo sexual y los indicadores de estrés nutricional en la población infanto-juvenil y adulta. Algunos de estos estudios también fueron pioneros porque introdujeron variables neurales y faciales al estudiar el crecimiento y el dimorfismo sexual. Los resultados obtenidos revelaron modificaciones alométricas en el crecimiento neural, y el facial presentó mayor tamaño; modificaciones que fueron explicadas aludiendo al modo de subsistencia de estas poblaciones, fundamentalmente cazadoras-recolectoras, y al estilo de vida que se deriva de dicha estrategia de subsistencia. Asimismo, se observó un marcado dimorfismo sexual entre los sujetos adultos y seniles, que coexistía con una menor estatura respecto de los tehuelches históricos. Además, toda la población mostró elevadas prevalencias de hipoplasia del esmalte dental y apiñamiento dentario, indicadores de estrés ambiental que, junto con los otros cambios observados, se asociaron a la pobreza en la que vivían esas poblaciones.

Héctor, junto con Walter Neves y otros investigadores de Brasil, en 2005, proyectaron los objetivos perseguidos en los estudios antes mencionados hacia tres comunidades Caboclo de isla de Marajó (Estado de Pará, Brasil). Los resultados obtenidos les permitieron confirmar el llamado "modelo latinoamericano" de desnutrición, es decir, baja prevalencia de retraso de crecimiento y emaciación, con predominio del primero sobre el segundo. En síntesis, las tres comunidades presentaban algún tipo de estrés nutricional, que constituyó la posible explicación para dar cuenta de los resultados obtenidos. 
También junto con Walter Neves, Héctor abordó una problemática siempre demandante del quehacer científico en el campo de la antropología biológica: el poblamiento americano. Todo comenzó con su estadía en el museo paranaense "Emilio Goeldi", en la ciudad de Belem, Brasil, cuando el interés se focalizó en el estudio de la variabilidad morfológica de los primates amazónicos actuales. Así, juntos iniciaron una investigación que reconoce ese antecedente y se orienta hacia el análisis del origen de los primeros americanos. Problemática que Héctor solía presentar en cada reunión científica con preguntas tales como: ¿quiénes son los amerindios? ¿Cómo llegaron a América? ¿Por dónde entraron? Preguntas que, aún hoy, permanecen sin respuestas conclusivas y convocan al debate en busca de su satisfacción.

Las observaciones de Walter y Héctor constituyeron uno de los modelos teóricos que, a partir de la década de 1990, pusieron en crisis al modelo Clovis, en la medida que sus explicaciones y escenarios acerca del poblamiento americano no se correspondían con la complejidad observada. Por ejemplo, mientras ese modelo, con la influencia de Aleš Hrdlička, sostenía que el pueblo Clovis revelaba un parentesco directo con poblaciones asiáticas, con características homogéneas, ahora esa población comenzaba a revelarse heterogénea, con patrones de variabilidad morfológica y lingüística que debían ser explicados. A partir de reanalizar la colección de cráneos humanos datados del Holoceno temprano de América (11.700-10.000 años AP) de Lagoa Santa, Brasil, aplicando técnicas de morfometría geométrica, pudieron demostrar que esos restos óseos no presentan rasgos mongoloides. Sus características son afines a aquellas presentes en grupos melanesio-australianos y africanos actuales. Por ello, llegaron a la conclusión de que la variación craneana observada a lo largo del tiempo en Sudamérica permite sostener la idea de que el Nuevo Mundo fue ocupado por dos o más poblaciones morfológicamente diferentes. Así, expusieron un nuevo modelo de poblamiento, llamado "Modelo de dos Componentes Biológicos Principales", según el cual los primeros americanos, paleoamericanos, tenían una morfología craneana que revelaría una ancestralidad con pueblos aborígenes actuales de Australia y África subsahariana y habrían entrado hace aproximadamente 14.000 años. Adicionalmente, sugieren una segunda migración hacia América, proveniente del noreste asiático, hace unos 11.000 años, cuyo patrón de morfología craneofacial revela vinculaciones con poblaciones mongoles actuales. En la actualidad, los estudios sobre poblamiento americano se orientan según una metodología interdisciplinaria, en la que convergen la genética de poblaciones, la paleoecología, la lingüística, la arqueología, la antropología biológica, entre otras, y que pone énfasis más en las causas de las diferencias observadas que en las mismas diferencias. En este sentido, aun cuando entre dichos estudios no existe consenso respecto de la explicación más probable para el origen de la diversidad biológica en América, los estudios de Héctor y Walter continúan siendo un referente imprescindible para el abordaje del tema.

Así, con su investigación antropobiológica, Héctor constituyó una escuela en la UNLP en la que, bajo su dirección y/o codirección, se formaron numerosos investigadores, quienes a su vez hoy prosiguen esa tarea en diversas instituciones universitarias de nuestro país.

Es preciso señalar que la vida de Héctor no transitó solo la docencia y la investigación; su compromiso con la Universidad reformista, cogobernada, lo llevó a ocupar diferentes cargos en la gestión de la FCNyM de la UNLP, así como a participar en actividades propias de ese modelo universitario. En el período 1986-1988 fue jefe del Consejo Consultivo y del Claustro de Profesores 
de Antropología. Participó, desde 1986, en las comisiones de investigaciones científicas de Grado Académico y de Plan de Estudios asesoras del Consejo Académico. Fue consejero académico durante cuatro períodos entre 1992 y 2004; y también, presidente de la Comisión de Investigaciones Científicas de la UNLP en el período 2001-2004.

Y un día de 1991 Héctor volvió al Museo de Ciencias Naturales como jefe, por concurso ordinario, de la División Antropología de la FCNyM. Por medio de su gestión supo equilibrar las funciones propias de un museo universitario, a saber, conservación, exhibición y extensión, con las de investigación y docencia. En este sentido, promovió la formación de recursos humanos por medio de la incorporación de becarios y tesistas al mencionado espacio institucional. Asimismo, desde esa función tuvo un rol decisivo en el desarrollo de las políticas de restitución de restos humanos a sus comunidades de origen. Muchas veces compartimos valiosos e interesantes debates acerca de redefinir el objeto de los estudios culturales: de la identidad a la heterogeneidad y la hibridación multiculturales. Sostenía que en la situación actual, de un mundo interconectado, donde los miembros de cada etnia, clase y nación se apropian de los repertorios heterogéneos de bienes y mensajes disponibles en los circuitos transnacionales, se generan nuevas formas de segmentación. Por ello, promovió que estudiar procesos culturales era, más que afirmar una identidad autosuficiente, conocer formas de situarse en medio de la heterogeneidad y de entender cómo se producen las hibridaciones. En este contexto, de intrincados debates, en 1994, mediante la aplicación de la ley № 23.940/91 - cuya autoría corresponde al senador por Chubut Hipólito Solari Irigoyen -, se produjo la primera restitución realizada en Argentina, la de los restos del cacique Inacayal, que fueron trasladados a Esquel para luego ser depositados por la comunidad en Tecka, lugar de origen del cacique.

Como parte de su labor profesional, Héctor tuvo una participación relevante en instituciones académicas y científicas nacionales e internacionales. Fue en 1991, junto con otros antropólogos biólogos, fundador de la Asociación de Antropología Biológica (AABA) y Director de la Revista Argentina de Antropología Biológica, órgano oficial de dicha asociación, desde 1994. Asimismo, se destacó como árbitro en prestigiosas revistas nacionales y extranjeras.

Los resultados de su prolífico trabajo de investigación se reflejaron en numerosas publicaciones en revistas nacionales e internacionales, en memorables debates posteriores a sus presentaciones en congresos, en capítulos de libros y en un libro, como único autor, titulado Evolución y diversificación biológica y humana desde la perspectiva craneofuncional, que en el año 2008 editara la Universidad Autónoma de México (Pucciarelli 2008).

A lo largo de su extensa vida profesional, recibió numerosos reconocimientos de diversas instituciones. Así, en 2010, la Universidad Nacional de La Plata le otorgó la distinción de Graduado Ilustre por sus aportes significativos a la antropología biológica de trascendencia nacional e internacional y por su desempeño académico y público. Asimismo, en oportunidad de la realización de las X y XII Jornadas Nacionales de la AABA, en 2011 y 2013 respectivamente, en una muestra de respeto, admiración y amistad, Héctor recibió sentidos reconocimientos a su trayectoria de Maestro de la Antropología Biológica, tal como la designación de Miembro Honorario de la AABA. Así también, en 2017, colegas y discípulos colaboraron en la redacción del libro De cómo cruzar fronteras en la ciencia (Pascheta et al. 2017)en su homenaje, cuya edición fue patrocinada por el Instituto Patagónico de Ciencias Sociales y Humanas y el 
Instituto de Investigaciones en Diversidad Cultural y Procesos de Cambio, ambos pertenecientes al CONICET. La fragilidad de su estado de salud, en esos momentos, le impidió recibir y disfrutar tan merecido homenaje.

Hasta aquí, hemos recreado la vida de Héctor sin hacer mención a Cristina, su esposa e infatigable compañera de prolongadas horas de trabajo en el laboratorio o en la división, ni a sus hijas, Damiana y Alejandra. Y la mención no responde a un mero imperativo formal, previsto para integrar un relato de vida, sino que constituye un factor fundamental que permite comprender cada una de las etapas de la vida de Héctor. Su esposa e hijas fueron el remanso y la fuerza necesarios para tomar las mejores decisiones ante disyuntivas cruciales, y así lo acompañaron durante toda su vida, hasta el final.

Por cierto, a modo de corolario del recuerdo de la vida de Héctor, en todos sus aspectos, corresponde afirmar que es casi imposible y doloroso encontrarle un final adecuado a tanta intensidad. Porque fue, además de un buen profesor, un Maestro, no solo por sus méritos profesionales, sino por su talento para promover, inspirar y orientar vocaciones hacia la docencia, la investigación y la extensión, pilares fundacionales de la Universidad pública.

Por ello, acudimos a la tierna melancolía con la que el poeta mexicano Amado Nervo sugirió recordar a los muertos queridos, a través del bello proloquio inglés: "Not Dead, But Gone Before": "No es que hayan muerto: se fueron antes".

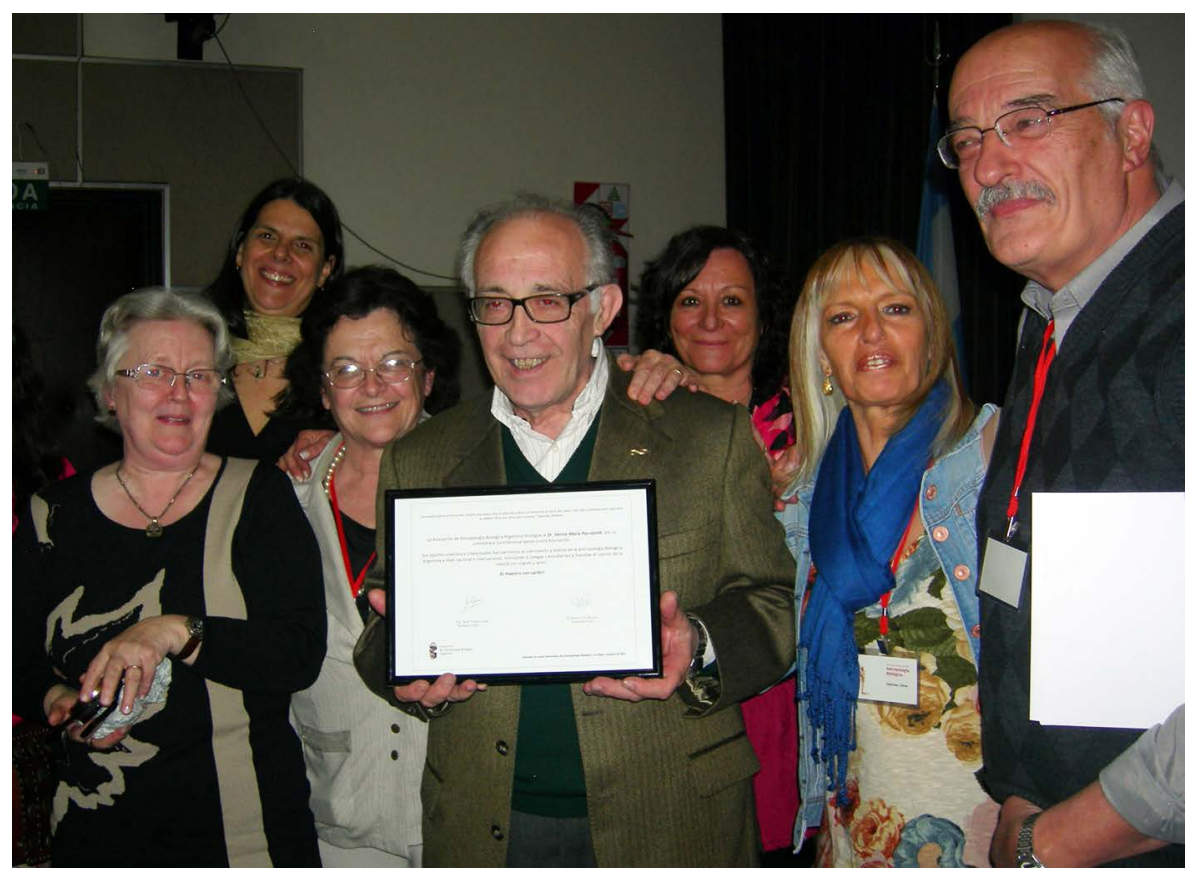

Fig. 2. Hector M. Pucciarelli recibe la distinción y el reconocimiento de la Asociación de Antropología Biológica Argentina AABA, año 2013. 


\section{Q Referencias bibliográficas}

»Anzelmo, M., Sardi, M. L., Barbeito-Andrés, J. y Pucciarelli, H. M. (2012). Alometrías ontogénicas y dimorfismo sexual facial en dos poblaciones humanas modernas. Revista Argentina de Antropología Biológica 14(1):89-100.

» Barbeito-Andrés, J., Pucciarelli, H. M. y Sardi, M. L. (2011). An ontogenetic approach to facial variation in three Native American populations. HOMO 62(1):56-67. DOI: 10.1016/j. jchb.2010.10.003

» Bernal, V., Perez, S. I., Gonzalez, P. N., Sardi, M. L. y Pucciarelli, H. M. (2010). Spatial patterns and evolutionary processes in southern South America: a study of dental morphometric variation. American Journal of Physical Anthropology 142(1):95-104. DOI: https:// doi.org/10.1002/ajpa.21206

»Carnese, F. R., Pucciarelli, H. M., Pinotti, L. V. y Dubois, C. M. F. (1994). Estándares de crecimiento normal en la población mapuche de Río Negro. Facultad de Filosofía y Letras de la Universidad de Buenos Aires. Serie Extensión Universitaria 1:1-51.

" Cesani, M. F., Orden, A. B., Oyhenart, E. E., Zucchi, M., Muñe, M. C y Pucciarelli, H. M. (2006). Growth of functional cranial components in rats submitted to intergenerational undernutrition. Journal of Anatomy 209(2):137-147. DOI: https://doi.org/10.1111/j.14697580.2006.00603.x

»Cesani, M. F., Oyhenart, E. E. y Pucciarelli, H. M. (2014). Effect of intergenerational chronic undernutrition on ponderal, and linear growth. ISRN Nutrition. Article ID 453460. DOI: $10.1155 / 2014 / 453460$

» Dahinten, S. L. y Pucciarelli, H. M. (1983). Effects of protein-calorie malnutrition during suckling and post-weaning periods on discontinuous cranial traits in rats. American Journal of Physical Anthropology 60:425-430. DOI: 10.1002/ajpa.1330600403

»Dahinten, S. L. y Pucciarelli, H. M. (1986). Variations in sexual dimorphism in the skulls of rats subjected to malnutrition, castration, and treatment with gonadal hormones. American Journal of Physical Anthropology 71:63-67. DOI: https://doi.org/10.1002/ ajpa.1330710108

"Dressino, V. y Pucciarelli, H. M. (1996). Efecto nutricional sobre el crecimiento craneofacial de Saimiri sciureus (Cebidae). Un estudio experimental. Revista Argentina de Antropología Biológica 1:98-112.

»Dressino, V. y Pucciarelli, H. M. (1997). Cranial growth in Saimiri sciureus (Cebidae) and its alteration by nutritional factors: A longitudinal study. American Journal of Physical Anthropology 102(4):545-554. DOI: https://doi.org/10.1002/(sici)10968644(199704)102:4<545::aid-ajpa8>3.0.c0;2-q

" Guimarey, L. M., Carnese, F. R., Pinotti, L. V., Pucciarelli, H. M. y Goicoechea, A. S. (1993). Crecimiento en escolares de Villa IAPI. Archivos Latinoamericanos de Nutrición 43: 139-145.

» González-José, R., Escapa, I., Neves, W. A., Cúneo, R. y Pucciarelli, H. M. (2008). Cladistic analysis of continuous modularized traits provides phylogenetic signal in Homo evolution. Nature 453:775-778. DOI: 10.1038/natureo6891.

» Luis, M. A., Lustig, A. L., Sardi, M. L., Ponce, P. V. y Pucciarelli, H. M. (1999). Posición de los araucanos en un contexto asiático-europeo. II: Metodología Howelliana. Revista Argentina de Antropología Biológica 2:187-200.

»Menéndez, L. P., Pucciarelli, H. M., Bonomo, M., Messineo, P. G., Gonzalez, M. E., Politis, 
G. G. y Perez, S. I. (2015). Early Holocene human remains from the Argentinean Pampas, cranial variation in South America and the American peopling. PaleoAmerica 1(3):251-265.

» Merton R. (1990). A hombros de gigantes. Ediciones Península. 218. pp. 97.

" Moss, M. L. y Young, R. W. (1960). A functional approach to craniology. American Journal of Physical Anthropology 18(4): 281-292. DOI: https://doi.org/10.1002/ajpa.1330180406

» Neves, W. A. y Pucciarelli, H. M. (1989). Extra continental biological relationships of early South American human remains: A multivariate analysis. Ciência e Cultura 41:566-575.

»Neves, W. A. y Pucciarelli, H. M. (1990). The origin of the first Americans: an analysis based on the cranial morphology of early South American human remains. American Journal of Physical Anthropology 81:274.

》Neves, W. A. y Pucciarelli, H. M. (1991). The origin of the first Americans: An analysis based on the cranial morphology of early South American human remains. Journal of Human Evolution 21:261-273. DOI: https://doi.org/10.1016/o047-2484(91)90107-7

» Orden, A. B., Pucciarelli., H. M., Muñe, M. C., Guimarey, L. M., Villanueva, M. E., Rodríguez, R. R. y Pons E. R. (1998). Efecto de las hormonas gonadales sobre el dimorfismo sexual en substrato de desnutrición. Acta Physiologica Pharmacologica et Therapeutica Latinoamericana 48(3):157-163.

» Oyhenart, E. E. y Pucciarelli, H. M. (1991). The influence of gonadic hormones on skull differences in rats malnourished during lactation. Acta Physiologica Pharmacologica et Therapeutica Latino Americana 41:287-293.

》 Oyhenart, E. E. y Pucciarelli, H. M. (1992). Sexual cranial dimorphism in malnourished rats treated with growth hormone. Growth, Development and Aging 56:179-184.

" Oyhenart, E. E., Torres, M. F., Pucciarelli, H. M., Dahinten, S. L. y Carnese, F. R. (2000). Growth and sexual dimorphism in aborigines from Chubut (Argentina). I: Body analysis. Acta Medica Auxologica 32(2):105-113.

»Paschetta, C., González-José, R. y Lanata, J. L. (Comps.). (2017). De cómo cruzar fronteras en la ciencia. Homenaje a Héctor M. Pucciarelli. Puerto Madryn y San Carlos de Bariloche: IPCSH e IIDyPCa, CONICET.

»Perez, S. I., Lema, V., Diniz-Filho, J. A. F., Bernal, V., González, P., Gobbo, D. y Pucciarelli, H. M. (2011). The role of diet and temperature in shaping cranial diversification of South American human populations: an approach based on spatial regression and rate tests. Journal of Biogeography 38:148-163. DOI: https://doi.org/10.1111/.1365-2699.2010.02392.x

"Pucciarelli, H. M. (1971). Variaciones craneanas en grupos raciales aborígenes de la República Argentina. Tesis Doctoral inédita. Facultad de Ciencias Naturales y Museo, UNLP, La Plata, Argentina. DOI: https://doi.org/10.25085/rsea.770402

» Pucciarelli, H. M. (1973). Experimental technique for cranial deformation in growing rats. Acta Physiologica Latino Americana 23(2):141-147.

》 Pucciarelli, H. M. (1974a). El método experimental en Antropología Biológica. Etnía 19:1-7.

»Pucciarelli, H. M. (1974b). The influence of experimental deformation on neurocranial wormian bones in rats. American Journal of Physical Anthropology 41(1):29-37. DOI: https://doi.org/10.1002/ajpa.1330410105

» Pucciarelli, H. M. (1978). The influence of experimental deformation on craniofacial development in rats. American Journal of Physical Anthropology 48:455-462.

»Pucciarelli, H. M. (1980). The effects of race, sex, and nutrition on craniofacial differentiation in rats. A multivariate analysis. American Journal of Physical Anthropology 53:359368. DOI: https://doi.org/10.1002/ajpa.1330530307 
» Pucciarelli, H. M. (1981). Growth of the functional components of the rat skull and its alteration by nutritional effects. A multivariate analysis. American Journal of Physical Anthropology 56:33-41. DOI: https://doi.org/10.1002/ajpa.1330560104

» Pucciarelli, H. M. (1989). Contribución al concepto de Antropología Biológica. Revista de Antropología 7:27-31.

» Pucciarelli, H. M. (1991). Nutrición y morfogénesis craneofacial. Una contribución de la Antropología Biológica Experimental. Interciencia 16(5):248-253.

»Pucciarelli, H. M. (2006). Dr. Melvin L. Moss, 1923-2006. Revista Argentina de Antropología Biológica 8(1):7-8.

» Pucciarelli, H. M. (2007). Aporte del método craneo-funcional a la discusión del modelo migración-reemplazo sobre poblamiento humano moderno. Arqueología Suramericana 3(1):84-102.

» Pucciarelli, H. M. (2008). Evolución y diversificación biológica humana desde la perspectiva craneofuncional. México DF: Universidad Autónoma de México.

» Pucciarelli, H. M. (2009). Explicando el Paleoamericano y su probable extinción, a través de inferencias comparativas y experimentales. RUNA XXX (1):9-27.

»Pucciarelli, H. M. y Oyhenart, E. E. (1987a). Effects of maternal food restriction during lactation on craniofacial growth in weanling rats. American Journal of Physical Anthropology 72:67-75. DOI: https://doi.org/10.1002/ajpa.1330720109

»Pucciarelli, H. M. y Oyhenart, E. E. (1987b). Influence of food restriction during gestation on craniofacial growth of the weanling rat. Acta Anatomica 129:182-187. DOI: https://doi. org/10.1159/000146397

» Pucciarelli, H. M., Oyhenart, E. E. y Terreros, M. C. (1984). Variations of rat skull bone robusticity evoked by malnutrition. American Journal of Physical Anthropology 64:119-124. DOI: https://doi.org/10.1002/ajpa.1330640204

» Pucciarelli, H. M., Moss, M. L., Shakaj, R., Moss-Salentijn, L., Sen, K. y Vilmann, H. (1987). Aplicación del método de los elementos finitos en los estudios de diferenciación craneofacial. Revista del Museo de La Plata. Nueva Serie. Tomo IX. Sección Antropología. № 66:1-20. DOI: https://doi.org/10.4995/thesis/10251/32957

» Pucciarelli, H. M., Dressino, V. y Niveiro, M. H. (1990). Changes in skull components of the squirrel monkey evoked by growth and nutrition: An experimental study. American Journal of Physical Anthropology 81:535-543. DOI: https://doi.org/10.1002/ajpa.1330810409

» Pucciarelli, H. M., Carnese, F. R., Pinotti, L. V., Guimarey, L. M. y Goicoechea, A. S. (1993). Sexual dimorphism in schoolchildrem of the Villa IAPI neighborhood (Quilmes, Buenos Aires, Argentina). American Journal of Physical Anthropology 92:165-172. DOI: https://doi. org/10.1002/ajpa.1330920206

» Pucciarelli, H. M., Sardi, M. L., Luis, M. A., Lustig, A. L., Ponce, P. V., Zanini, M. C. y Neves, W. A. (1999). Posición de los araucanos en un contexto asiático-europeo. I: Metodología craneofuncional. Revista Argentina de Antropología Biológica 2:163-185.

» Pucciarelli, H. M., Neves, W. A., Melcher, S. S. y Murrieta, R. S. S. (2005). Nutritional status and sexual dimorphism in three amazonian caboclo communities. Anthropologie $X L I I I / 1: 63-75$.

»Pucciarelli, H. M., González-José, R., Neves, W. A., Sardi, M. L. y Ramírez Rozzi, F (2008). East-West cranial differentiation in pre-columbian populations from Central and North America. Journal of Human Evolution 54:296-308. DOI: https://doi.org/10.1016/j.jhevol.2007.08.011 
»Pucciarelli, H. M., Perez, S. I. y Politis, G. G. (2010). Early Holocene human remains from the Argentinean Pampas: Additional evidence for distinctive cranial morphology of early South Americans. American Journal of Physical Anthropology 143(2):298-305. DOI: https://doi.org/10.1002/ajpa.21347

»Sardi, M. L. y Pucciarelli, H. M. (200o). Posición relativa de fueguinos y araucanos en un contexto intercontinental. Un estudio multivariado de craneología funcional. Anales del Instituto de la Patagonia. Serie Ciencias Humanas 28:73-8o. DOI: https://doi.org/10.4067/ s0718-686×2016000100oo6

» Torres, M. F., Oyhenart, E. E., Dahinten, S. L., Carnese, F. R. y Pucciarelli, H. M. (2000). Growth and sexual dimorphism in aborigines from Chubut (Argentina). II: Head and face analysis. Acta Medica Auxologica 32(2):115-123. 
